\title{
Adaptación de la multidimensional sportspersonship orientations scale al contexto español de la educación física
}

\section{Adaptation of the multidimensional sportspersonship orientations scale to the spanish physical education context}

\author{
Rafael Burgueño ${ }^{1}$, Isabel Sánchez-Gallardo² y Jesús Medina-Casaubón ${ }^{2}$ \\ ${ }^{1}$ Departamento de Educación. Universidad de Almería \\ ${ }^{2}$ Departamento de Educación Física y Deportiva. Universidad de Granada
}

Resumen: El objetivo fue analizar la estructura factorial de las diferentes versiones propuestas para la Multidimensional Sportspersonship Orientations Scale (MSOS) en el contexto espańol de la educación física. Los participantes fueron 652 estudiantes de educación secundaria y bachillerato (353 hombres y 299 mujeres; $M_{\text {edad }}=14.98, D T_{\text {edad }}=1.59$ ). El análisis factorial confirmatorio no apoyó psicométricamente ninguna de las versiones planteadas por los trabajos previos para la MSOS. Sin embargo, los hallazgos sustentaron psicométricamente un modelo de cuatro factores correlacionados y 17 ítems (i.e., respecto por las convenciones sociales, respeto por las reglas, compromiso y respeto por el compañero) para la educación física. Esta estructura fue invariante por género. Las correlaciones sustentaron la validez discriminante del instrumento. El análisis de fiabilidad reflejó valores adecuados para cada factor. Como conclusión, se recomienda la utilización de una nueva estructural factorial para la MSOS en el contexto espańol de la educación física.

Palabras clave: Conducta ética, educación en valores, deportividad, pedagogía de la educación física, propiedades psicométricas.
Abstract: The objective was to analyse the factor structure of the different versions proposed for the Multidimensional Sportspersonship Orientations Scale (MSOS) in the Spanish Physical Education context. The participants were 652 Post-Compulsory and Compulsory Secondary Education students (353 men y 299 women; $M_{\text {age }}=14.98, S D_{\text {age }}=1.59$ ). Confirmatory factor analysis psychometrically supported none of the different versions prosed by the previous works for the MSOS. Nevertheless, the findings underpinned a 17 -items 4 -factor model (i.e., respect for social conventions, respect for rules and teacher, commitment and respect for classmates) for Physical Education. This structure was invariant across gender. The correlations supported the instrument's discriminant validity. Reliability analysis reflected suitable values for each factor. In its conclusion, the use of a new factor structure for the MSOS was recommended in the Spanish Physical Education context.

Keywords: Ethical behaviour, values education, sportspersonship, Physical Education pedagogy, psychometric properties.

\section{Introducción}

En los últimos años, hay un incremento en el número de investigaciones que tratan de abordar el estudio de la ética y la moral en el deporte como un medio para combatir tanto los comportamientos agresivos y violentos (Bermejo, Borrás, Haces-Soutullo, \& Ponseti, 2018; Borrueco, Angulo, Viladrich, Pallarés, \& Cruz, 2018) como el dopaje (Chantal $\&$ Bernache-Assollant, 2017), que se producen con mayor frecuencia entre los deportistas. En este contexto, el constructo de deportividad puede constituir una nueva aproximación conceptual que permita comprender, de manera más precisa, el desarrollo de la conducta ética en el deportista en edad escolar (Iturbide-Luquin \& Elosua-Oliden, 2017; Shrout, Voelker, Munro, \& Kubitz, 2017). Durante la etapa de escolarización del deportista, la Educación Física parece representar un adecuado vehículo a la hora de favorecer la educación ética y en valores en el estudiante y, en consecuencia, con-

Dirección para correspondencia [Correspodence address]: Isabel Sánchez Gallardo. Facultad de Ciencias del Deporte, Universidad de Granada. Carretera de Alfacar, s/n, 18071, Granada (España). E-mail: isabelsg@ ugr.es tribuir al desarrollo de la deportividad del individuo (Kirk, 2013; Yukhymenko-Lescroart, 2018).

Tradicionalmente, la investigación ha profundizado esencialmente en la identificación y comprensión de los determinantes fundamentales de las conductas de deportividad (Monaci, De Palo, \& Sinatra, 2014). En base a esto, se han distinguido tres aproximaciones teóricas a la hora de abordar el análisis de la deportividad. La primera de ellas, la perspectiva del aprendizaje-social postula que las experiencias vicarias y de refuerzo concurren para moldear las percepciones del individuo sobre lo que representa la conducta apropiada o inapropiada dentro de los diferentes escenarios deportivos (Bandura, 1986). El segundo enfoque es la aproximación desarrollo-estructural, la cual relaciona el refuerzo de la conducta de deportividad con el desarrollo moral de los jóvenes deportistas (Haan, 1983). El tercer modelo conceptual es la visión socio-psicológica desarrollada por Vallerand y colaboradores (Vallerand, Deshaies, Cuerrier, Brière, \& Pelletier, 1996; Vallerand \& Losier, 1994).

De acuerdo con esta visión socio-psicológica, la deportividad puede ser conceptualizada de manera multidimensional 
mediante cinco dimensiones: a) respeto por las convenciones sociales, b) respeto por las reglas y jueces, c) compromiso con la participación deportiva, d) respeto por el oponente y, e) relativa visión negativa de participar en el deporte (Vallerand, Brière, Blanchard, \& Provencher, 1997). De esta manera, la deportividad se traduciría en varias dimensiones u orientaciones dentro de la mente de todo deportista. Además, se hipotetiza que los atletas generalmente se comportarían de acuerdo con su respaldo relativo a estas cinco dimensiones de deportividad (Vallerand et al., 1997, 1996).

Con la finalidad de medir el constructo de deportividad, Vallerand et al. (1997) desarrollaron la Multidimensional Sportspersonship Orientations Scale (MSOS). Este instrumento es un autoinforme compuesto por 25 ítems agrupados en 5 factores, pese a obtener un valor marginal de consistencia interna para visión negativa $(\alpha=.57)$, el análisis psicométrico sustentó empíricamente esta estructura factorial. Este modelo de cinco factores y 25 ítems fue respaldado en el contexto tanto griego (Proios, Doganis, \& Proios, 2006) como español (Martín-Albo, Núñez, Navarro-Izquierdo, \& González-Ruiz, 2006), aunque Martín-Albo et al. (2006) informaron de la necesidad de correlacionar cuatro pares de error (MSOS-25b).

Por otra parte, un conjunto de trabajos cuestionaron la estructura factorial propuesta inicialmente por Vallerand et al. (1997), encontrando modelos factoriales plausibles con cierto apoyo psicométrico. Concretamente, una estructura de cuatro factores correlacionados y 20 ítems (MSOS-4F) fue sostenida en deportistas turcos (Sezen-Balcikanli, 2014), franceses (Chantal, Robin, Vernat, \& Bernache-Assollant, 2005), noruegos (Miller, Roberts, \& Ommundsen, 2004) y suecos (Lemyre, Roberts, \& Ommundsen, 2002) después de la eliminación del factor visión negativa. En este sentido, McCutcheon (1999) sugirió que los problemas relativos al factor visión negativa podrían derivarse de una ausencia de consistencia interna entre los cinco ítems de este factor y, por ende, consideran que éstos no capturan el sentido de ganar a toda costa que trata de reflejar este factor.

En cambio, Jing-Horng y Hsu (2015) apoyaron una estructura de tres factores correlacionados y 18 ítems (MSOS3F), compuestos por ocho ítems para el factor compromiso y respeto por las reglas, por seis ítems para el factor respeto por las convecciones social y oponentes y, cuatro ítems para el factor visión negativa en deportistas taiwaneses. Mientras que trabajos como Gano-Overway, Guivernau, Magyar, Waldron, y Ewing (2005), Bofarull y Cusí (2014) o Lamoneda-Prieto, Huertas-Delgado, Córdoba-Caro, y García (2014) respaldaron un modelo de dos factores correlacionados (MSOS-2F) en deportistas estadounidenses y españoles, respectivamente.

A la luz de los resultados inconsistentes relacionados con las propiedades psicométricas de la MSOS, junto con la ausencia de un instrumento con la sensibilidad necesaria en la medición de la deportividad en el contexto español de la EF; el objetivo fue analizar la estructura factorial de las versiones propuestas para la MSOS (Vallerand et al., 1997) con alumnado de EF de educación secundaria obligatoria y bachillerato. Una vez definida la mejor estructura, un objetivo secundario fue examinar la invariancia factorial por género, así como la consistencia interna y la estabilidad temporal del instrumento.

\section{Material y Método}

\section{Participantes}

Los participantes fueron 652 estudiantes de educación secundaria obligatoria y de bachillerato (353 hombres y 299 mujeres), con una edad comprendida entre 12 y 19 años $\left(M_{\text {edad }}=14.98, D T_{\text {edad }}=1.59\right)$, quienes realizaban educación física en centros educativos públicos de una ciudad situada en el sureste español. Para analizar la estabilidad temporal del instrumento, tomaron parte 45 estudiantes de primero de bachillerato (22 hombres y 23 mujeres; $M_{\text {edad }}=16.32, D T_{\text {edad }}=$ $0.57)$, quienes completaron el instrumento de medida en dos ocasiones con un intervalo de tiempo de 6 semanas entre la primera y segunda toma de datos.

\section{Instrumentos}

Orientaciones a la Deportividad en EF. Se utilizó la versión española (Martín-Albo et al., 2006) Multidimensional Sportspersonship Orientations Scale (Vallerand et al., 1997) adaptada a la EF. El instrumento consta de 25 ítems agrupados en 5 ítems por factor para juzgar el respeto por las convenciones sociales, respeto por las reglas y por el profesor, compromiso, respeto por los compañeros de clase y, visión negativa. Cada ítem se responde mediante una escala Likert de 5 puntos, desde 1 (no), punto medio 3 (a veces), a 5 (sí).

\section{Diseño y Procedimiento}

La presente investigación fue un diseño instrumental, puesto que pretende analizar la psicometría de un instrumento de medición (Ato, López-García, \& Benavente, 2013). El proceso de adaptación de la escala fue realizado de acuerdo con las directrices propuestas por Muñiz, Elosua, y Hambleton (2013). Primero, un grupo de cuatro expertos en EF y deporte adaptó cada uno de los ítems que componen la versión espańola en el deporte de la Multidimensional Sportspersonship Orientations Scale al dominio de la EF. Para tal finalidad, la palabra "adversario" fue sustituida por "compañero/a de clase", "decisiones arbitrales" por "decisiones del profesor/a", "entrenamientos" por "clase de Educación Física", "competición" por "actividades/ juegos". Segundo, un nuevo grupo de cuatro expertos en EF y Deporte revisaron dicha versión para verificar tanto la adecuada comprensión como 
la objetividad de los contenidos de los 25 ítems que integran el instrumento. Tercero, se realizó un estudio piloto con un grupo de estudiantes de educación secundaria $(N=10)$ con la finalidad de comprobar la correcta comprensión de los ítems y estimar un tiempo promedio para la administración del instrumento. En su conjunto, los resultados del proceso de adaptación ofrecieron evidencias de validez basada en el contenido del instrumento.

A continuación, el personal investigador contactó con el equipo directivo y con el profesorado de EF de los diferentes centros educativos participantes para solicitar su colaboración. La escala fue administrada por el personal investigador quien explicó que la participación era voluntaria y anónima. Durante la administración del cuestionario, el personal investigador estuvo a disposición de los encuestados para resolver todas las dudas aparecidas durante este proceso.

\section{Análisis Estadístico}

Se utilizaron los programas estadísticos SPSS v.19 y AMOS v.19 para llevar a cabo el tratamiento estadístico de los datos. La validez de la estructura interna del instrumento fue analizada mediante el análisis factorial confirmatorio para cada uno de los modelos factoriales propuestos por la literatura. Para efectuar la técnica del análisis factorial confirmatorio, se seleccionó el método de máxima verosimilitud con el procedimiento bootstrapping de 5000 re-muestreos (Kline, 2011), dada la violación del supuesto de normalidad multivariante (coeficiente de Mardia $=93.85, p<.01$; coeficiente de Mardia $=80.04, p<.01$; coeficiente de Mardia = 70.91, $p<.01$; coeficiente de Mardia $=60.21, p<.01$; coeficiente de Mardia $=66.13, p<.01)$. La bondad del ajuste fue juzgada por medio de una serie de índices de ajuste: ratio chi-cuadrado y grados de libertad $\left(\chi^{2} / g l\right)$, Comparative Fit Index (CFI), TuckerLewis Index (TLI), Standardized Root Mean Square Residual (SRMR), Root Mean Square Error of Approximation (RMSEA) con su intervalo de confianza al 90\% y, Akaike Information Criteria (AIC). Kline (2011) propone valores menores que 3 para $\chi^{2} / g l$, mayores que .95 para CFI y TLI, así como menores que .080 para SRMR y .050 para RMSEA como representativos de un buen ajuste. El AIC se utiliza como criterio para comparar modelos competidores, indicando que el menor valor obtenido por este parámetro representaría el modelo con mejor ajuste y parsimonia (Kline, 2011). Los pesos de regresión estandarizados son aceptables con valores superiores a .50 , mientras que las covarianzas residuales estandarizadas son adecuadas con valores absolutos menores que 2.54 (Hair, Black, Babin, \& Anderson, 2010). Las correlaciones entre factores muestran una adecuada discriminación conceptual con valores menores que .85 (Kline, 2011).

Para realizar el análisis multi-grupo de invariancia factorial por género, se siguió el enfoque metodológico avanzado por Gregorich (2006). En este aspecto, la disminución en los valores de CFI y RMSEA inferiores a .010 y .015, respectivamente, entre los sucesivos modelos restringidos apoyaría la invariancia factorial del instrumento (Chen, 2007). Para analizar la consistencia interna del instrumento, se estimaron el coeficiente alfa de Cronbach $(\alpha)$ y el coeficiente de fiabilidad compuesta de Raykov $(\rho)$, aceptables con valores mayores que 70 (Viladrich, Angulo-Brunet, \& Doval, 2017). Para examinar la estabilidad temporal del instrumento, se determinó el coeficiente de correlación intraclase (CCI) junto con su intervalo de confianza al 95\% (IC 95\%), satisfactorio con valores superiores a 70 (Koo \& Li, 2016).

\section{Resultados}

\section{Análisis Factorial Confirmatorio}

La Tabla 1 muestra los resultados obtenidos para cada una de las versiones identificadas por la literatura previa para la MSOS. Estas versiones (MSOS-25, MSOS-25b, MSOS-4F, y MSOS-3F) revelaron una pobre bondad del ajuste reflejada en valores de $\chi^{2} / g l$ mayores que 3 , menores que .95 para CFI y TLI, incluso menores que .90 para estos índices de la MSOS-25 y MSOS-2F, y valores superiores a .050 para RMSEA. El análisis de modificación de índices informó, en su conjunto, de ciertos errores en la estructura interna para cada versión. La inspección de modificación de índices mostró, en su conjunto, determinados errores en la estructura interna de cada versión. En concreto, se obtuvieron valores absolutos mayores que 2.54 concernientes a las covarianzas residuales estandarizadas para la MSOS-25 (ítem 4, ítem 5, ítem 10, ítem 15, ítem 20, ítem 24 e ítem 25), MSOS-25b (ítem 5, ítem 10, ítem 15, ítem 20, ítem 25), MSOS-4F (ítem 4 e ítem 9), MSOS-3F (ítem 4, ítem 13, ítem 20 e ítem 25), así como para la MSOS-2F (ítem 4, ítem 7, ítem 8, ítem 17 e ítem 18).

Tomando como punto de partida el análisis de la estructura interna de la versión original del instrumento (MSOS-25) planteada por Vallerand et al. (1997), se decidió descartar aquellos ítems con covarianzas residuales estandarizadas superiores al valor absoluto 2.54. Como consecuencia de esto, se efectuó un análisis factorial confirmatorio para testar la sostenibilidad de un modelo de cuatro factores y 16 ítems después de eliminar el ítem 4, ítem 5, ítem 10, ítem 15, ítem 20, ítem 24 e ítem 25 (MSOS-EF). Como refleja la Tabla 1, la MSOS-EF obtuvo índices de ajuste apropiados, además del mejor ajuste con los datos entre los diferentes modelos testados. Es más, esta estructura factorial obtuvo el menor valor AIC, siendo, por consiguiente, la más parsimoniosa. En su conjunto, las evidencias sugieren que la MSOS-EF tuvo un mejor funcionamiento psicométrico respecto a las demás propuestas testadas. Por esta serie de argumentos, se utilizó esta versión del instrumento para los restantes análisis psicométricos. 
Tabla 1. Índices de Ajuste para cada Modelo Factorial propuesto para la MSOS.

\begin{tabular}{lccccccccc}
\hline & $\chi^{2}$ & $g l$ & $\chi^{2} / g l$ & $C F I$ & TLI & SRMR & RMSEA (IC90\%) & AIC \\
\hline MSOS-25 & $811.41^{* * *}$ & 265 & 3.06 & .899 & .885 & .060 & $.056(.052, .062)$ & 932.409 \\
MSOS-25b & $712.70^{* * *}$ & 260 & 2.74 & .916 & .903 & .056 & $.052(.047, .056)$ & 842.702 \\
MSOS-4F & $514.73^{* * *}$ & 164 & 3.13 & .927 & .915 & .045 & $.057(.052, .063)$ & 606.725 \\
MSOS-3F & $502.37^{* * *}$ & 143 & 3.81 & .910 & .896 & .052 & $.066(.060, .072)$ & 580.355 \\
MSOS-2F & $740.64^{* * *}$ & 118 & 6.28 & .833 & .808 & .067 & $.090(.084, .096)$ & 810.640 \\
MSOS-EF & $277.06^{* * *}$ & 112 & 2.48 & .960 & .952 & .032 & $.048(.041, .055)$ & 359.055 \\
\hline
\end{tabular}

Nota: MSOS-25 = versión original; MSOS-25b = versión con cuatro correlaciones de pares de error; MSOS-4F = versión de cuatro factores y 20 ítems; MSOS-3F = versión de tres factores y 18 ítems; MSOS-2F = versión de dos factores y 18 ítems; MSOS-EF = versión de cuatro factores y 17 ítems para la Educación Física.

$* * * p<.001$

La Figura 1 muestra los pesos de regresión estandarizados para la totalidad de los ítems, los cuales oscilaron entre $.57 \mathrm{y}$ $.79 \mathrm{y}$, fueron estadísticamente significativos $(p<.001)$. Las covarianzas residuales estandarizadas estuvieron comprendidas entre -1.98 y 2.24. Las correlaciones entre factores oscilaron entre .51 y .66 .

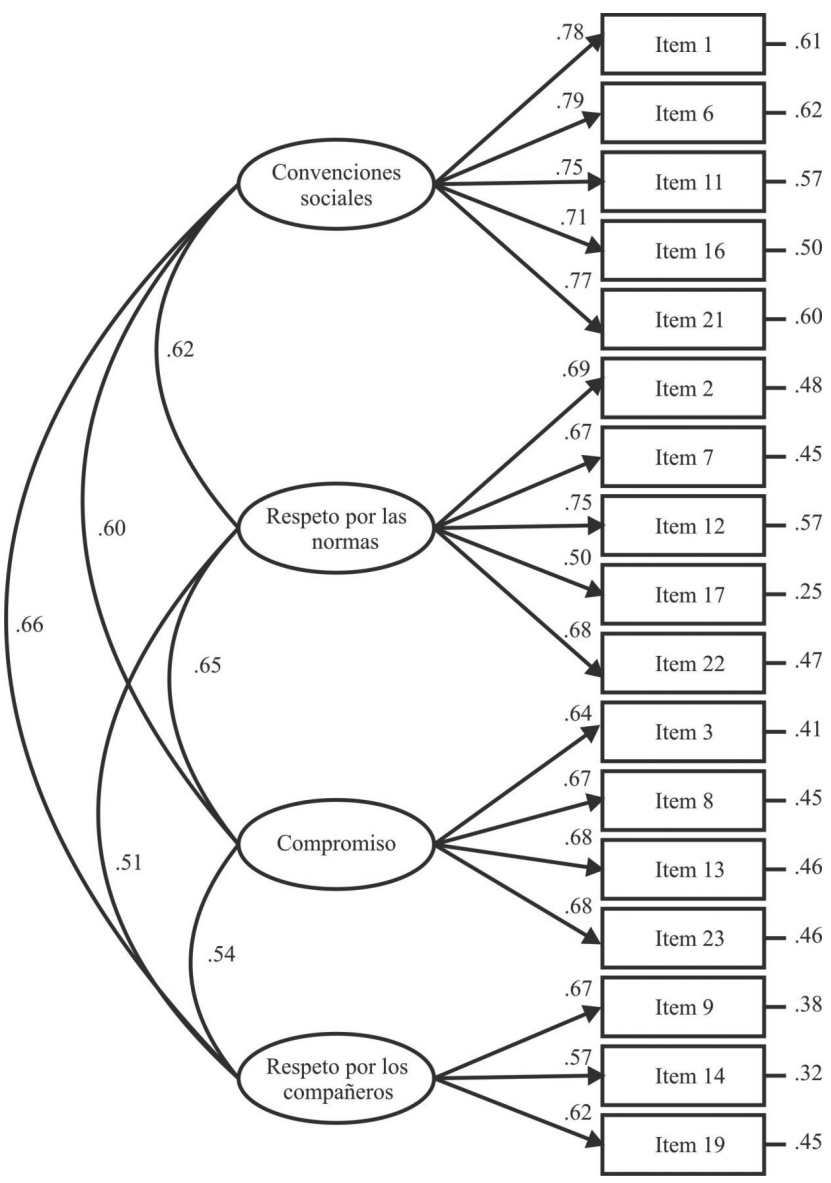

Figura 1. Análisis Factorial Confirmatorio de la Escala Multidimensional en Orientaciones a la Deportividad en Educación Física.

Las elipses representan los factores latentes, mientras que los rectángulos simbolizan los respectivos ítems del instrumento.

\section{Análisis de Invariancia Factorial por Género}

La Tabla 2 muestra respectivamente incrementos inferiores a .010 para el valor CFI, así como, incrementos menores que .015 para el valor RMSEA entre cada uno de los cuatro modelos progresivamente restringidos. Por tanto, la hipótesis nula de invariancia factorial por género no se puede rechazar. 
Tabla 2. Análisis Multi-Grupo de Invarianza Factorial por Género.

\begin{tabular}{|c|c|c|c|c|c|c|c|c|c|c|c|c|}
\hline & $\chi^{2}$ & $g l$ & $\chi^{2} / g l$ & CFI & $T L I$ & $S R M R$ & RMSEA (IC90\%) & $C M$ & $\Delta \chi^{2}$ & $\Delta g l$ & $\triangle C F I$ & $\triangle R M S E A$ \\
\hline 1. Invariancia configural & 435.53 & 224 & 1.94 & .948 & .937 & .039 & $.038(.033, .044)$ & - & - & - & - & - \\
\hline 2. Invariancia métrica & 455.70 & 237 & 1.92 & .946 & .938 & .041 & $.038(.033, .043)$ & 2 vs. 1 & 20.18 & 13 & -.002 & .000 \\
\hline 3. Invariancia fuerte & 510.82 & 254 & 2.01 & .937 & .932 & .042 & $.040(.035, .045)$ & 3 vs. 2 & $55.11^{* * *}$ & 17 & -.009 & .002 \\
\hline 4. Invariancia estricta & 551.54 & 271 & 2.04 & .931 & .930 & .042 & $.040(.035, .045)$ & 4 vs. 3 & $40.72^{* * *}$ & 17 & -.006 & .000 \\
\hline
\end{tabular}

Nota: $\mathrm{CM}=$ Comparación de Modelos.

${ }^{* * *} p<.01,{ }^{* *} p<.01,{ }^{*} p<.05$

\section{Estadísticos Descriptivos y Fiabilidad}

La Tabla 3 muestra valores promedios, a excepción del factor visión negativa, superiores al punto medio para cada uno de los factores respecto a su escala de medida. De igual manera, los valores de consistencia interna fueron adecuados para cada uno factores que componen este instrumento tanto para alfa de Cronbach como para el coeficiente de fiabilidad compuesta de Raykov. Los valores de estabilidad temporal mostraron un valor aceptable para cada uno de los cuatros factores del instrumento (CCI > .70).

Tabla 3. Estadísticos Descriptivos y Coeficientes de Fiabilidad.

\begin{tabular}{|c|c|c|c|c|c|c|c|c|c|}
\hline & \multicolumn{6}{|c|}{$N=652$} & \multirow[b]{2}{*}{$\alpha$} & \multirow[b]{2}{*}{$\rho$} & \multirow{2}{*}{$\begin{array}{c}N=45 \\
\text { CCI (IC 95\%) }\end{array}$} \\
\hline & Rango & $M$ & $D T$ & Asimetría & Curtosis & Varianza & & & \\
\hline Convenciones Sociales & $1-5$ & 4.01 & 0.80 & -0.73 & 0.46 & 0.64 & .87 & .87 & $.78(.65, .85)$ \\
\hline Respeto por las reglas & $1-5$ & 4.22 & 0.61 & -0.82 & 0.81 & 0.37 & .76 & .80 & $.75(.69, .82)$ \\
\hline Compromiso & $1-5$ & 4.14 & 0.69 & -0.75 & 0.37 & 0.48 & .76 & .76 & $.73(.59, .81)$ \\
\hline Respeto por los compańeros & $1-5$ & 3.71 & 0.89 & -0.51 & 0.09 & 0.78 & .71 & .72 & $.70(.58, .83)$ \\
\hline
\end{tabular}

Nota: $\alpha=$ Alfa de Cronbach; $\rho$ = Coeficiente de Fiabilidad Compuesta de Raykov; CCI = Coeficiente de Correlación Intraclase.

${ }^{* * *} p<.001,{ }^{* *} p<.01,{ }^{*} p<.05$

\section{Discusión}

El objetivo fue analizar la estructura factorial de las versiones propuestas para la MSOS (Vallerand et al., 1997) con alumnado de EF de educación secundaria obligatoria y bachillerato. Una vez definida la mejor estructura, un objetivo secundario fue examinar la invariancia factorial por género, así como la consistencia interna y la estabilidad temporal del instrumento. Los resultados obtenidos sustentan la utilización de una nueva estructural factorial compuesta por 4 factores y 17 ítems en lugar de los diferentes modelos propuestos por la investigación previa para la EF.

Los resultados del análisis factorial confirmatorio destacan la idoneidad de una nueva estructura de 4 factores correlacionados y 17 ítems (i.e., respeto por las convenciones sociales, respeto por las reglas y profesor, compromiso y respeto por los compañeros) en alumnado de EF en educación secundaria obligatoria y bachillerato. En este punto, los resultados de este trabajo se encuentran alineados con aquellos estudios que defendieron desechar el factor visión negativa dado su bajo rendimiento psicométrico (Chantal et al., 2005; Lemyre et al., 2002; Miller et al., 2004; Sezen-Balcikanli, 2014). En esta misma línea, estos hallaz- gos comparten el planteamiento realizado por McCutcheon (1999), en la medida de que los cinco ítems que componen el factor visión negativa no consiguieron captar y medir el significado que este factor pretende representar y, de ahí, la necesidad de su eliminación. Es más, Kirk (2013) apunta que las propensiones propias de la EF como al búsqueda de la cooperación, la colaboración, el compañerismo o la valoración del esfuerzo entre el alumnado, hace pensar que la visión negativa no tuviera cabida en este contexto. Por otra parte, las correlaciones provenientes del análisis factorial confirmatorio mostraron valores similares a aquellos encontrados por la investigación previa (Martín-Albo et al., 2006; Miller et al., 2004; Vallerand et al., 1997). Dichas puntuaciones moderadas dan sustento empírico a la divergencia conceptual entre constructo y, por tanto, ofrecen evidencias a la validez discriminante del instrumento.

Los resultados del análisis multi-grupo de invariancia factorial por género proporcionaron evidencias que respaldaron el carácter invariante de la MSOS-EF en alumnado de EF de secundaria obligatoria y bachillerato.

Los hallazgos de esta investigación ofrecieron evidencias que apoyaron la invariancia factorial en función del género para la MSOS-EF, lo cual se muestra en línea con la investi- 
gación previa. En este aspecto, los resultados son de gran interés puesto que apoyan la utilización en Espańa de la MSOSEF para examinar las posibles diferencias concernientes a la orientación a la deportividad entre alumnos y alumnas en clase de EF.

Por otra parte, el análisis de consistencia interna mostró valores aceptables para cada uno de las cuatro factores que integran la MSOS-EF, lo cual está delineado con los valores obtenidos por la investigación previa en el ámbito deportivo (Lemyre et al., 2002; Martín-Albo et al., 2006; Sezen-Balcikanli, 2014; Vallerand et al., 1996). De igual modo, no se encontraron problemas de consistencia interna reflejados previamente para el factor respeto por los oponentes/ compañeros (Miller et al., 2004). Asimismo, estos hallazgos referidos a la consistencia interna del instrumento son novedosos puesto que suministran evidencias que apuntalan la fiabilidad compuesta del instrumento, hecho del que hasta este momento no se tenía constancia. El análisis de estabilidad temporal arrojó valores adecuados para cada uno de los factores, lo cual se muestra en consonancia con investigaciones previas (MartínAlbo et al., 2006; Vallerand et al., 1997).

A pesar de los resultados obtenidos en el actual trabajo, ciertas limitaciones deben ser mencionadas. La muestra participante corresponde con una muestra de conveniencia, lo que hace interpretar los resultados con cautela y, por tanto, no puedan ser generalizados al conjunto de la población estudiantil. En este aspecto, se precisan nuevos estudios que examinen la estructura factorial defendida en esta investigación en otros entornos de actividad deportiva (i.e., deporte de formación, deporte de rendimiento), así como en otros entornos socioculturales. De igual forma, futuras investigaciones deben abordar las limitaciones del actual estudio referidas al contenido de los ítems de la visión negativa y su relación con el concepto de deportividad. Asimismo, sería necesario verificar la asociación predictiva entre la MSOS-EF y otro tipo de variables con la finalidad de probar la validez de criterio de este instrumento.

La presente investigación proporciona evidencias que aconsejan el uso de una nueva estructura factorial para la MSOS (i.e., modelo de 4 factores correlacionados y 17 ítems) en el alumnado de EF de educación secundaria obligatoria y bachillerato con la finalidad de medir el constructo de deportividad. Este avance permitirá conocer la posible influencia de las clases de EF sobre el desarrollo del sentido de deportividad del alumnado y, analizar si esta deportividad desarrollada en clase de EF podría tener una cierta transferencia al comportamiento del estudiante como deportista en el ámbito deportivo. De esta manera, se podría determinar el impacto de las clases EF como un instrumento para tratar de erradicar las conductas disruptivas en el ámbito deportivo.

\section{Apéndice}

Convenciones Sociales

1. Cuando pierdo, felicito a mi compañero/a sea quien sea.

6. Tras perder una actividad, doy la mano a mi compañero/a

11. Tras una actividad, felicito a mi compañero/a de clase por su actuación

16. Después de ganar una actividad, reconozco el buen juego de mi compañero/a

21. Gane o pierda, doy la mano a mi compañero/a.

Respeto a las reglas y al profesorado

2. Respeto las decisiones del profesor/a.

7. Respeto las reglas.

12. Realmente respeto todas las normas de la clase.

17. Respeto las decisiones del profesor/a aunque estén equivocadas.

22. Respeto una decisión aunque no provenga del profesor/a.

Compromiso

3. Cuando participo en clase de Educación Física, me entrego por completo.

8. No me rindo, ni después de cometer mucho errores.

13. Busco maneras de mejorar mis puntos débiles.
18. Considero importante asistir a clase de Educación Física.*

23. Durante las clases de Educación Física, me entrego por completo.

\section{Respeto por el compañero/a}

4. Ayudo a mi compañero/a a levantarse tras una caída.*

9. Si puedo, pido al profesor/a que permita a mi compañero/a seguir jugando en caso de que haya sido eliminado injustamente.

14. Cuando un compañero/a se lesiona, pido al profesor/a que detenga la actividad para poder asistirlo.

19. Si veo que un compañero/a es castigado o sancionado injustamente, intento que el profesor/a rectifique su decisión.

24. Si un compańero/a olvida su ropa deportiva, yo le presto la que tengo de repuesto.*

Visión negativa

5. Participo en Educación Física por el honor personal y la nota.*

10. Critico lo que el profesor/a me obliga a hacer.*

15. Después de una actividad, busco excusas para justificar mi comportamiento.* 
20. Cuando el profesor/a señala los errores que he cometido después de una actividad, me niego a admitirlos.*
25. Si cometo un error durante una actividad, me enfado. *

* Ítem eliminado para la versión en Educación Física.

\section{Referencias bibliográficas}

1. Ato, M., López-García, J. J., \& Benavente, A. (2013). A classification system for research designs in psychology. Annals of Psychology, 29(3), 1038-1059. http://doi.org/10.6018/analesps.29.3.178511

2. Bandura, A. (1986). Social foundations of thought and action: A social cognitive theory. Englewood Cliffs, NJ: Prentice-Hall.

3. Bermejo, J. M., Borrás, P. A., Haces-Soutullo, M. V., \& Ponseti, F. J. (2018). Is fair play losing value in grassroots sport? Revista de Psicologia Del Deporte, 27(3), 1-4.

4. Bofarull, I., \& Cusí, M. (2014). Sportspersonship in school and extracurricular sports. Apunts. Educación Física Y Deportes, 116(2), 52-59. http://doi.org/http://dx.doi.org/10.5672/apunts.2014-0983. es. $(2014 / 2) .116 .05$

5. Borrueco, M., Angulo, A., Viladrich, C., Pallarés, S., \& Cruz, J. (2018). Clima motivacional, orientación a la deportividad y predisposición al engaño en futbolistas jóvenes. Revista de Psicologia Del Deporte, 27(3), 10-17.

6. Chantal, Y., \& Bernache-Assollant, I. (2017). Yellow and social perceptions of racing cyclists' sportspersonship: Proposing an inter-contextual analysis. Journal of Sports Sciences, 35(6), 525-530. http://doi.org/10.10 80/02640414.2016.1175654

7. Chantal, Y., Robin, P., Vernat, J. P., \& Bernache-Assollant, I. (2005). Motivation, sportspersonship, and athletic aggression: A mediational analysis. Psychology of Sport and Exercise, 6(2), 233-249. http://doi. org/10.1016/j.psychsport.2003.10.010

8. Chen, F. F. (2007). Sensitivity of goodness of fit indexes to lack of measurement invariance. Structural Equation Modeling, 14(3), 464-504. http://doi.org/10.1080/10705510701301834

9. Gano-Overway, L. A., Guivernau, M., Magyar, T. M., Waldron, J. J., \& Ewing, M. E. (2005). Achievement goal perspectives, perceptions of the motivational climate, and sportspersonship: Individual and team effects. Psychology of Sport and Exercise, 6(2), 215-232. http://doi. org/10.1016/j.psychsport.2003.11.001

10. Gregorich, S. E. (2006). Do self-report instruments allow meaningful comparisons across diverse population groups? Testing measurement invariance using the confirmatory factor analysis framework. Medical Care, 44(11 Suppl 3), S78-S94. http://doi.org/10.1097/01. mlr.0000245454.12228.8f

11. Haan, N. (1983). An interactional morality of everyday life. In N. Haan, R. Bellah, P. Rabinow, \& W. Sullivan (Eds.), Social science as moral inquiry (pp. 218-250). New York, NY: Columbia University Press.

12. Hair, J. F. J., Black, W. C., Babin, B. J., \& Anderson, R. E. (2010). Multivariate data analysis (7th ed.). Englewood Cliffs, NJ: Pearson Prentice Hall.

13. Iturbide-Luquin, L.-M., \& Elosua-Oliden, P. (2017). The values associated with the sport: Analysis and evaluation of sportspersonship. Revista de Psicodidáctica, 22(1), 29-36. http://doi.org/10.1387/RevPsicodidact. 15918

14. Jing-Horng, F., \& Hsu, Y. (2015). The interaction between paternalistic leadership and achievement goals in predicting athletes' sportspersonship. Kinesiology, 47(1), 115-122.

15. Kirk, D. (2013). Educational value and models-based practice in Physical Education. Educational Philosophy and Theory, 45(9), 973-986. http://doi.org/10.1080/00131857.2013.785352

16. Kline, R. B. (2011). Principles and practice of structural equation modeling (3rd ed.). New York, NY: The Guilford Press. http://doi. org $/ 10.1038 / 156278 \mathrm{a} 0$
17. Koo, T. K., \& Li, M. Y. (2016). A guideline of selecting and reporting intraclass correlation coefficients for reliability research. Journal of Chiropractic Medicine, 15(2), 155-163. http://doi.org/10.1016/j. jcm.2016.02.012

18. Lamoneda-Prieto, J., Huertas-Delgado, F. J., Córdoba-Caro, L. G., \& García, V. (2014). Adaptation of the multidimensional sportspersonship orientation scale in juvenile football. Cuadernos de Psicología Del Deporte, 14(2), 71-80.

19. Lemyre, P.-N., Roberts, G. C., \& Ommundsen, Y. (2002). Achievement goal orientations, perceived ability, and sportspersonship in youth soccer. Journal of Applied Sport Psychology, 14(2), 120-136. http://doi. org/10.1080/10413200252907789

20. Martín-Albo, J., Núńez, J. L., Navarro-Izquierdo, J. G., \& GonzálezRuiz, V. M. (2006). Validity of the Spanish version of the multidimensional sportpersonship orientations scale. Revista de Psicología Del Deporte, 15(1), 9-22.

21. McCutcheon, L. E. (1999). The multidimensional sportspersonship orientations scale has psychometric problems. Journal of Social Behavior \& Personality, 14(3), 439-444.

22. Miller, B. W., Roberts, G. C., \& Ommundsen, Y. (2004). Effect of motivational climate on sportspersonship among competitive youth male and female football players. Scandinavian Journal of Medicine and Science in Sports, 14(3), 193-202. http://doi.org/10.1111/j.16000838.2003.00320.x

23. Monaci, L., De Palo, V., \& Sinatra, M. (2014). Sportpersonship behaviours: An exploratory investigation of antecedents. International Journal of Sport Psychology, 45(3), 231-245. http://doi.org/10.7352/ IJSP2014.45.231

24. Muñiz, J., Elosua, P., \& Hambleton, R. K. (2013). International test commission guidelines for test translation and adaptation: Second edition. Psicothema, 25(2), 151-157. http://doi.org/10.7334/psicothema2013.24

25. Proios, M., Doganis, G., \& Proios, M. (2006). Form of athletic exercise, school environment, and sex in development of high school students' sportsmanship. Perceptual and Motor Skills, 103(1), 96-196. http://doi. org/10.2466/pms.103.1.99-106

26. Sezen-Balcikanli, G. (2014). The Turkish adaptation of multidimensional sportspersonship orientation scale-MSOS: A reliability and validity study. Gazi Journal of Physical Education and Sports Science, 15(1), 1-10.

27. Shrout, R. M., Voelker, D. K., Munro, G. D., \& Kubitz, K. A. (2017). Associations between sport participation, goal and sportspersonship orientations, and moral reasoning. Ethics \& Behavior, 27(6), 502-518. http://doi.org/10.1080/10508422.2016.1233494

28. Vallerand, R. J., Brière, N. M., Blanchard, C., \& Provencher, P. (1997). Development and validation of the multidimensional sportspersonship orientations scale. Journal of Sport and Exercise Psychology, 19(2), 197206. http://doi.org/10.1123/jsep.19.2.197

29. Vallerand, R. J., Deshaies, P., Cuerrier, J.-P., Brière, N. M., \& Pelletier, L. G. (1996). Toward a multidimensional definition of sportsmanship. Journal of Applied Sport Psychology, 8(1), 89-101. http://doi. org/10.1080/10413209608406310

30. Vallerand, R. J., \& Losier, G. F. (1994). Self-determined motivation and sportsmanship orientations: An assessment of their temporal relationship. Journal of Sport \& Exercise Psychology, 16(3), $229-245$. 
31. Viladrich, C., Angulo-Brunet, A., \& Doval, E. (2017). A journey around alpha and omega to estimate internal consistency reliability. Annals of Psychology, 33(3), 755-782. http://doi.org/10.6018/analesps.33.3.268401
32. Yukhymenko-Lescroart, M. A. (2018). On identity and sport conduct of student-athletes: Considering athletic and academic contexts. Psychology of Sport and Exercise, 34, 10-19. http://doi.org/10.1016/j.psychsport.2017.09.006 